\title{
MAPPING PROPERTIES OF BASIC HYPERGEOMETRIC FUNCTIONS
}

\author{
ÁRPÁD BARICZ AND ANBHU SWAMINATHAN
}

\begin{abstract}
It is known that the ratio of Gaussian hypergeometric functions can be represented by means of $g$-fractions. In this work, the ratio of $q$-hypergeometric functions are represented by means of $g$-fractions that lead to certain results on $q$-starlikeness of the $q$-hypergeometric functions defined on the open unit disk. Corresponding results for the $q$-convex case are also obtained.
\end{abstract}

Mathematics subject classification (2010): 33D15, 33C05, 30C45.

Keywords and phrases: Continued fractions, Pick functions, hypergeometric functions, starlike functions, convex functions, $q$-hypergeometric functions, quotients of hypergeometric functions.

\section{REFERENCES}

[1] S. AgRawal and S. Sahoo, Geometric properties of basic hypergeometric functions, J. Difference Equ. Appl. 20 (2014), 1502-1522.

[2] S. AgRawal AND S. K. SAhoo, A generalization od starlike functions of order alpha, arXiv.1404.3988.

[3] N. I. AKHIEZER, The classical moment problem and some related questions in analysis, Translated by N. Kemmer Hafner Publishing Co., New York, 1965.

[4] G. E. Andrews, R. Askey And R. Roy, Special functions, Encyclopedia of Mathematics and its Applications, 71, Cambridge Univ. Press, Cambridge, 1999.

[5] Á. Baricz, K. Raghavendar and A. Swaminathan, Turán type inequalities for $q$ hypergeometric functions, J. Approx. Theory 168 (2013), 69-79.

[6] C. Berg And H. L. Pedersen, Pick functions related to the gamma function, Rocky Mountain J. Math. 32 (2002), 507-525.

[7] C. Berg AND H. L. Pedersen, A completely monotone function related to the gamma function, J. Comp. Appl. Math. 133 (2001), 219-230.

[8] W. F. Donoghue, JR., The interpolation of Pick functions, Rocky Mountain J. Math. 4 (1974), 169173.

[9] P. L. DuREn, Univalent functions, Grundlehren der Mathematischen Wissenschaften, 259, Springer, New York, 1983.

[10] G. Gasper And M. Rahman, Basic hypergeometric series, Cambridge Univ. Press, Cambridge, 1990.

[11] D. P. Gupta, M. E. H. Ismail And D. R. Masson, Contiguous relations, basic hypergeometric functions, and orthogonal polynomials. II. Associated big q-Jacobi polynomials, J. Math. Anal. Appl. 171 (1992), 477-497.

[12] P. HÄstÖ, S. Ponnusamy AND M. VuORInen, Starlikeness of the Gaussian hypergeometric functions, Complex Var. Elliptic Equ. 55 (2010), 173-184.

[13] E. HEINE, Untersuchunger über die Reihe, J. Reine Angew. Math. 34 (1847), 285-328.

[14] M. E. H. Ismail, E. Merkes And D. Styer, A generalization of starlike functions, Complex Variables Theory Appl. 14 (1990), 77-84.

[15] M. E. H. Ismail AND C. A. LiBIS, Contiguous relations, basic hypergeometric functions, and orthogonal polynomials. I, J. Math. Anal. Appl. 141 (1989), 349-372.

[16] M. E. H. Ismail And M. Rahman, The associated Askey-Wilson polynomials, Trans. Amer. Math. Soc. 328 (1991), 201-237. 
[17] W. B. Jones, O. Njåstad And W. J. Thron, Schur fractions, Perron Carathéodory fractions and Szegó polynomials, a survey, in Analytic theory of continued fractions, II (Pitlochry/Aviemore, 1985), 127-158, Lecture Notes in Math., 1199, Springer, Berlin.

[18] R. KÜSTNER, Mapping properties of hypergeometric functions and convolutions of starlike or convex functions of order $\alpha$, Comput. Methods Funct. Theory 2 (2002), 597-610.

[19] R. KÜstNeR, On the order of starlikeness of the shifted Gauss hypergeometric function, J. Math. Anal. Appl. 334 (2007), 1363-1385.

[20] L. Lorentzen And H. WaAdeland, Continued fractions with applications, Studies in Computational Mathematics, 3, North-Holland, Amsterdam, 1992.

[21] E. P. Merkes, On typically-real functions in a cut plane, Proc. Amer. Math. Soc. 10 (1959), 863-868.

[22] S. Ponnusamy, Close-to-convexity properties of Gaussian hypergeometric functions, J. Comput. Appl. Math. 88 (1997), 327-337.

[23] S. Ponnus Amy AND M. Vuorinen, Univalence and convexity properties for Gaussian hypergeometric functions, Rocky Mountain J. Math. 31 (2001), 327-353.

[24] K. RaghaVEndar AND A. SWAminathan, Close-to-convexity properties of basic hypergeometric functions using their Taylor coefficients, J. Math. Appl. 35 (2012), 53-67.

[25] F. RønNing, A. Szegó quadrature formula arising from q-starlike functions, in Continued fractions and orthogonal functions (Loen, 1992), 345-352, Dekker, New York.

[26] S. K. Sahoo And N. L. Sharma, On a generalization of close-to-convex functions, Ann. Polon. Math. 113 (2015), 93-108.

[27] H. S. WALL, Analytic Theory of Continued Fractions, D. Van Nostrand Company, Inc., New York, N. Y., 1948. 